\title{
RADIUS OF STARLIKENESS FOR SOME CLASSES CONTAINING NON-UNIVALENT FUNCTIONS
}

\author{
SHALU YADAV, KANIKA SHARMA, AND V. RAVICHANDRAN
}

\begin{abstract}
A starlike univalent function $f$ is characterized by the function $z f^{\prime}(z) / f(z)$; several subclasses of these functions were studied in the past by restricting the function $z f^{\prime}(z) / f(z)$ to take values in a region $\Omega$ on the right-half plane, or, equivalently, by requiring the function $z f^{\prime}(z) / f(z)$ to be subordinate to the corresponding mapping of the unit disk $\mathbb{D}$ to the region $\Omega$. The mappings $w_{1}(z):=z+\sqrt{1+z^{2}}, w_{2}(z):=\sqrt{1+z}$ and $w_{3}(z):=e^{z}$ maps the unit disk $\mathbb{D}$ to various regions in the right half plane. For normalized analytic functions $f$ satisfying the conditions that $f(z) / g(z), g(z) / z p(z)$ and $p(z)$ are subordinate to the functions $w_{i}, i=1,2,3$ in various ways for some analytic functions $g(z)$ and $p(z)$, we determine the sharp radius for them to belong to various subclasses of starlike functions.
\end{abstract}

\section{InTRODUCTION}

Though complex numbers is not ordered field, the inequalities in the real line can be extended to complex plane in a natural way using the concept of subordination. Let $f, g$ be two analytic functions defined on the open unit disk $\mathbb{D}:=\{z \in \mathbb{C}:|z|<1\}$. The function $f$ is subordinate to the function $g$, written $f \prec g$, if $f=g \circ w$ for some analytic function $w: \mathbb{D} \rightarrow \mathbb{D}$ with $w(0)=0$ (and such a function $w$ is known as a Schwartz function). A univalent function $f: \mathbb{D} \rightarrow \mathbb{C}$ is always locally univalent or, in other words, it has nonvanishing derivative. Therefore, the study of univalent functions can be restricted to the functions normalized by $f(0)=f^{\prime}(0)-1=0$. We let $\mathcal{A}$ denote the class of all analytic functions $f: \mathbb{D} \rightarrow \mathbb{C}$ normalized by the conditions $f(0)=0$ and $f^{\prime}(0)=1$. Since $f^{\prime}(0) \neq 0$ for functions $f \in \mathcal{A}$, the functions in the class $\mathcal{A}$ are univalent in some disk centred at the origin. The largest disk centered at the origin in which $f$ is univalent is called as the radius of univalence of the function $f$. Consider a subset $\mathcal{M} \subset \mathcal{A}$ and a property $P$ (such as univalence, starlikeness, convexity) that the image of the functions in $\mathcal{M}$ may or may not have. It often happens that the image of $\mathbb{D}_{r}=\{z \in \mathbb{D}:|z|<r\}$ for some $r \leq 1$ has the property $P$; the largest $\rho_{f}$ such that the functions has the property $P$ in each disk $\mathbb{D}_{r}$ for $0<r \leq \rho_{f}$ is the radius of the property $P$ of the function $f$. The number $\rho:=\inf \left\{\rho_{f}: f \in \mathcal{M}\right\}$ is the radius of the property $P$ of the class $\mathcal{M}$; if $\mathcal{G}$ is the class of all $f \in \mathcal{A}$ characterized by the property $P$, then $\rho$ is called the $\mathcal{G}$-radius of the class $\mathcal{M}$. The $\mathcal{G}$-radius of the class $\mathcal{M}$ is denoted by $R_{\mathcal{G}}(\mathcal{M})$ or simply by $R_{\mathcal{G}}$ if the class $\mathcal{M}$ is clear from the context.

The class $\mathcal{M}$ we are interested in is characterized by the ratio between functions $f$ and $g$ belonging to $\mathcal{A}$; several authors [1, 2, 6, 9, 10, 11, 15, 17] have studied such classes. The

2020 Mathematics Subject Classification. 30C80, 30C45.

Key words and phrases. Starlike functions; subordination; radius of starlikeness; lune.

The research of Shalu Yadav is supported by an institute fellowship from NIT Trichy. 
classes which we are considering are as follows.

$$
\begin{aligned}
\mathcal{T}_{1}=\left\{f \in \mathcal{A}: \frac{f}{g} \prec e^{z}, \frac{g}{z p} \prec e^{z} \quad \text { for some } \quad g \in \mathcal{A} \quad \text { and } \quad p \prec \sqrt{1+z}\right\} \\
\mathcal{T}_{2}=\left\{f \in \mathcal{A}: \frac{f}{g} \prec \sqrt{1+z}, \frac{g}{z p} \prec \sqrt{1+z} \quad \text { for some } \quad g \in \mathcal{A} \quad \text { and } \quad p \prec e^{z}\right\} \\
\mathcal{T}_{3}=\left\{f \in \mathcal{A}: \frac{f}{g} \prec z+\sqrt{1+z^{2}}, \frac{g}{z p} \prec z+\sqrt{1+z^{2}} \quad \text { for some } g \in \mathcal{A}\right. \text { and } \\
\left.p \prec z+\sqrt{1+z^{2}}\right\} .
\end{aligned}
$$

These classes are motivated by a recent work of Ali, Sharma and Ravichandran [?] wherein similar classes were investigated. These classes contain non-univalent functions and this makes the study of such functions interesting. We compute $\mathcal{G}$-radius when $\mathcal{G}$ is one of the following subclasses of starlike functions studied recently in the literature. A starlike univalent function $f$ is characterized by the condition $\operatorname{Re}\left(z f^{\prime}(z) / f(z)\right)>0$. If we define the class $\mathcal{P}$ as the class of all functions $p(z)=1+c_{1} z+\cdots$ defined on $\mathbb{D}$ satisfying $\operatorname{Re} p(z)>0$, it follows that a function $f$ is starlike if and only if $z f^{\prime}(z) / f(z) \in \mathcal{P}$. Several subclasses of starlike functions were studied in the past by restricting the function $z f^{\prime}(z) / f(z)$ to take values in a region $\Omega$ on the right-half plane, or, equivalently, by requiring the function $z f^{\prime}(z) / f(z)$ to be subordinate to the corresponding mapping $\varphi: \mathbb{D} \rightarrow \Omega: z f^{\prime}(z) / f(z) \prec$ $\varphi(z)$. For $\varphi(z)=(1+(1-2 \alpha) z) /(1-z), 0 \leq \alpha<1$, this class is the class $\mathcal{S}^{*}(\alpha)$ of all starlike functions of order $\alpha$. Several other subclasses of starlike functions are defined by replacing the superordinate functions $\varphi$ by functions having nice geometry. For the functions $\varphi$ defined by $\varphi(z):=(1+A z) /(1+B z)$, with $-1 \leq B<A \leq 1, e^{z}, 1+(4 / 3) z+$ $(2 / 3) z^{2}, 1+\sin z, z+\sqrt{1+z^{2}}, 1+\left(z k+z^{2}\right) /\left(k^{2}-k z\right)$ where $k=\sqrt{2}+1,1+(2(\log ((1+$ $\left.\sqrt{z}) /(1-\sqrt{z}))^{2} \pi^{2}\right), 2 /\left(1+e^{-z}\right)$ and $1+z-z^{3} / 3$, we denote the class of all functions $f \in \mathcal{A}$ with $z f^{\prime}(z) / f(z) \prec \varphi(z)$ respectively by $\mathcal{S}^{*}[A, B], \mathcal{S}_{\text {exp }}^{*}, \mathcal{S}_{c}^{*}, \mathcal{S}_{\text {sin }}^{*}, \mathcal{S}_{\overparen{\zeta}}^{*} \mathcal{S}_{R}^{*}, \mathcal{S}_{p}^{*}, \mathcal{S}_{S G}^{*}$ and $\mathcal{S}_{N_{e}}^{*}$.

\section{The Radius of univalence}

2.1. Class $\mathcal{T}_{1}$. This class consists of the analytic functions $f$ such that $f(z) / g(z)$ is subordinate to $e^{z}$ and $p$ is subordinate to $\sqrt{1+z}$ for some analytic function $g$. This class is non-empty as the function $f_{1}: \mathbb{D} \rightarrow \mathbb{C}$, defined by

$$
f_{1}(z)=z e^{2 z} \sqrt{1+z}
$$

belongs to the class $\mathcal{T}_{1}$. The function $f_{1}$ satisfies the subordination condition for this class along with the functions $g_{1}, p_{1}: \mathbb{D} \rightarrow \mathbb{C}$ by

$$
g_{1}(z)=z e^{z} \sqrt{1+z} \text { and } p_{1}(z)=\sqrt{1+z} .
$$

The function $f_{1}$ plays the role of extremal for this class. Since

$$
f_{1}^{\prime}(z)=\frac{e^{2 z}\left(4 z^{2}+7 z+2\right)}{2 \sqrt{1+z}},
$$

it is clear that $f^{\prime}(-7+\sqrt{17} / 8)=0$, and so the radius of univalence is at most $(-7+\sqrt{17}) / 8$. As the function $f_{1}$ is not univalent in $\mathbb{D}$, the class $\mathcal{T}_{1}$ contains non-univalent functions. Here also we find that radius of univalence and the radius of starlikeness for $\mathcal{T}_{1}$ are same and is $(-7+\sqrt{17}) / 8 \approx-0.359612$. 
To do this, we need to first find a disk into which the disk $\mathbb{D}_{r}$ is mapped by the function $f \in \mathcal{T}_{1}$. Let $f \in \mathcal{T}_{1}$ and define the functions $p_{1}, p_{2}$ by $p_{1}(z)=f(z) / g(z)$ and $p_{2}(z)=$ $g(z) / z p(z)$. Then $f(z)=z p(z) p_{1}(z) p_{2}(z)$ and

$$
\left|\frac{z f^{\prime}(z)}{f(z)}-1\right| \leq\left|\frac{z p^{\prime}(z)}{p(z)}\right|+\left|\frac{z p_{1}^{\prime}(z)}{p_{1}(z)}\right|+\left|\frac{z p_{2}^{\prime}(z)}{p_{2}(z)}\right| .
$$

Using the bounds for $p, p_{1}, p_{2}$, we obtain

$$
\left|\frac{z f^{\prime}(z)}{f(z)}-1\right| \leq\left\{\begin{array}{l}
\frac{r(5-4 r)}{2(1-r)} ; \quad r \leq \sqrt{2}-1 \\
\frac{3+r+7 r^{2}+3 r^{4}}{2\left(1-r^{2}\right)} ; r \geq \sqrt{2}-1 .
\end{array}\right.
$$

for each function $f \in \mathcal{T}_{1}$. Clearly, for $|z|=r \leq(-7+\sqrt{17}) / 8$, we have

$$
\left|\frac{z f^{\prime}(z)}{f(z)}-1\right| \leq \frac{r(5-4 r)}{2(1-r)} \leq 1 \text {. }
$$

This shows that the radius of starlikeness is at least $(-7+\sqrt{17}) / 8$. Since the radius of univalence is atmost $(-7+\sqrt{17}) / 8$, it follows that the radius univalence and radius of starlikeness are both equal to $(-7+\sqrt{17}) / 8$.

2.2. Class $\mathcal{T}_{2}$. Functions of this class are analytic functions $f$ satisfying the subordinations

$$
\frac{f(z)}{g(z)} \prec \sqrt{1+z}, \quad \frac{g(z)}{z p(z)} \prec \sqrt{1+z} \quad \text { and } \quad p(z) \prec e^{z},
$$

where the functions $g \in \mathcal{A}$ and $p \in \mathcal{P}$.

This class is non-empty as the function $f_{2}: \mathbb{D} \rightarrow \mathbb{C}$ defined by

$$
f_{2}(z)=z(1+z) e^{z}
$$

belongs to the class $\mathcal{T}_{2}$. The function $f_{2}$ satisfies the required subordinations when we define the functions $g_{2}, p_{2}: \mathbb{D} \rightarrow \mathbb{C}$ by

$$
g_{2}(z)=z e^{z} \sqrt{1+z} \text { and } \quad p_{2}(z)=e^{z} .
$$

This function $f_{2}$ plays the role of extremal for this class. Since

$$
f_{2}^{\prime}(z)=e^{z}\left(1+3 z+z^{2}\right)
$$

it is clear that $f_{2}^{\prime}(-3+\sqrt{5} / 2)=0$, and so the radius of univalence is atmost $(-3+\sqrt{5}) / 2$. We shall show that the radius of univalence and the radius of starlikeness for $\mathcal{T}_{2}$ are equal and commom value of the radius is precisely $(-3+\sqrt{5}) / 2 \approx-0.381966$.

To do this, we need to first find a disk into which the disk $\mathbb{D}_{r}$ is mapped by the function $f \in \mathcal{T}_{2}$. Let $f \in \mathcal{T}_{2}$ and define the functions $p_{1}, p_{2}$ by $p_{1}(z)=f(z) / g(z)$ and $p_{2}(z)=$ $g(z) / z p(z)$. Then $f(z)=z p(z) p_{1}(z) p_{2}(z)$ and

$$
\left|\frac{z f^{\prime}(z)}{f(z)}-1\right| \leq\left|\frac{z p^{\prime}(z)}{p(z)}\right|+\left|\frac{z p_{1}^{\prime}(z)}{p_{1}(z)}\right|+\left|\frac{z p_{2}^{\prime}(z)}{p_{2}(z)}\right| .
$$

Using the bounds for $p, p_{1}, p_{2}$, we obtain

$$
\left|\frac{z f^{\prime}(z)}{f(z)}-1\right| \leq\left\{\begin{array}{l}
\frac{r(-2+r)}{(-1+r)} ; \quad r \leq \sqrt{2}-1 \\
\frac{1+4 r+6 r^{2}+r^{4}}{4\left(1-r^{2}\right)} ; r \geq \sqrt{2}-1 .
\end{array}\right.
$$

for each function $f \in \mathcal{T}_{2}$. Clearly, for $|z|=r \leq(-3+\sqrt{5}) / 2$, we have 


$$
\left|\frac{z f^{\prime}(z)}{f(z)}-1\right| \leq \frac{r(-2+r)}{(-1+r)} \leq 1
$$

This shows that the radius of starlikeness is at least $(-3+\sqrt{5}) / 2$. Since the radius of univalence is atmost $(-3+\sqrt{5}) / 2$, it follows that the radius univalence and radius of starlikeness are both equal to $(-3+\sqrt{5}) / 2$.

2.3. Class $\mathcal{T}_{3}$. Recall that a function $f \in \mathcal{A}$ belongs the class $\mathcal{T}_{3}$ if there are functions $g \in \mathcal{A}$ and $p \in \mathcal{P}$ satisfying the subordinations

$$
\frac{f(z)}{g(z)} \prec z+\sqrt{1+z^{2}}, \quad \frac{g(z)}{z x p(z)} \prec z+\sqrt{1+z^{2}} \quad \text { and } \quad p(z) \prec z+\sqrt{1+z^{2}} \text {. }
$$

This class is non-empty as the function $f_{3}: \mathbb{D} \rightarrow \mathbb{C}$ defined by

$$
f_{3}(z)=z\left(z+\sqrt{1+z^{2}}\right)^{3}
$$

belongs to the class $\mathcal{T}_{3}$. Indeed, the function $f_{3}$ satisfies the required subordinations when we define the functions $g_{3}, p_{3}: \mathbb{D} \rightarrow \mathbb{C}$ by

$$
g_{3}(z)=z\left(z+\sqrt{1+z^{2}}\right)^{2} \text { and } \quad p_{3}(z)=z+\sqrt{1+z^{2}} .
$$

The function $f_{3}$ plays the role of extremal for this class. Since

$$
f_{3}^{\prime}(z)=\frac{\left(z+\sqrt{1+z^{2}}\right)^{3}\left(3 z+\sqrt{1+z^{2}}\right)}{\sqrt{1+z^{2}}}
$$

it is clear that $f_{3}^{\prime}(-1 / \sqrt{8})=0$, and so the radius of univalence is at most $1 / \sqrt{8}$. Also, since the function $f_{3}$ is not univalent in $\mathbb{D}$, the class $\mathcal{T}_{3}$ contains non-univalent functions. Indeed, we shall show that the radius of univalence and the radius of starlikeness for $\mathcal{T}_{3}$ are equal and the common value of the radius is precisely $1 / \sqrt{8} \approx 0.353553$.

To do this, we need to first find a disk into which the disk $\mathbb{D}_{r}$ is mapped by the function $f \in \mathcal{T}_{3}$. Let $f \in \mathcal{T}_{3}$ and define the functions $p_{1}, p_{2}$ by $p_{1}(z)=f(z) / g(z)$ and $p_{2}(z)=$ $g(z) / z p(z)$. Then $f(z)=z p(z) p_{1}(z) p_{2}(z)$ and

$$
\left|\frac{z f^{\prime}(z)}{f(z)}-1\right| \leq\left|\frac{z p^{\prime}(z)}{p(z)}\right|+\left|\frac{z p_{1}^{\prime}(z)}{p_{1}(z)}\right|+\left|\frac{z p_{2}^{\prime}(z)}{p_{2}(z)}\right| .
$$

For $p \in \mathcal{P}$ with $p(z) \prec z+\sqrt{1+z^{2}}$, Afis and Noor [4] have shown that

$$
|p(z)| \leq r+\sqrt{1+r^{2}}, \quad\left|\frac{z p^{\prime}(z)}{p(z)} \leq \frac{r}{\sqrt{1+r^{2}}}\right| \quad(|z| \leq r) .
$$

Using these inequalities for $p, p_{1}, p_{2} \prec z+\sqrt{1+z^{2}}$, we see that

$$
\left|\frac{z f^{\prime}(z)}{f(z)}-1\right| \leq \frac{3 r}{\sqrt{1+r^{2}}}, \quad|z| \leq r,
$$

for each function $f \in \mathcal{T}_{3}$. Clearly, for $|z|=r \leq 1 / \sqrt{8}$, we have

$$
\left|\frac{z f^{\prime}(z)}{f(z)}-1\right| \leq \frac{3 r}{\sqrt{1+r^{2}}} \leq 1 \text {. }
$$


This shows that the radius of starlikeness is at least $1 / \sqrt{8}$. Since the radius of univalence is at most $1 / \sqrt{8}$, it follows that the radius of univalence and the radius of starlikeness are both equal to $1 / \sqrt{8}$.

\section{RADIUS OF STARLIKENESS}

Our first theorem gives the sharp radius of starlikeness of order $\alpha$ of the classes $\mathcal{T}_{1}, \mathcal{T}_{2}$ and $\mathcal{T}_{3}$. We shall show that this radius is the same for the subclass $\mathcal{S}_{\alpha}^{*}$ consisting of all functions $f \in \mathcal{S}^{*}(\alpha)$ satisfying $\left|z f^{\prime}(z) / f(z)-1\right|<1-\alpha$.

Theorem 3.1. The following results hold for the classes $\mathcal{S}^{*}(\alpha)$ and $\mathcal{S}_{\alpha}^{*}$.

(i) $R_{\mathcal{S}^{*}(\alpha)}\left(\mathcal{T}_{1}\right)=R_{\mathcal{S}_{\alpha}^{*}}\left(\mathcal{T}_{1}\right)=\left(7-2 \alpha-\sqrt{17+4 \alpha+4 \alpha^{2}}\right) / 8$

(ii) $R_{\mathcal{S}^{*}(\alpha)}\left(\mathcal{T}_{2}\right)=R_{\mathcal{S}_{\alpha}^{*}}\left(\mathcal{T}_{2}\right)=\left(\left(3-\alpha-\sqrt{5-2 \alpha+\alpha^{2}}\right)\right) / 2$

(iii) $R_{\mathcal{S}^{*}(\alpha)}\left(\mathcal{T}_{3}\right)=R_{\mathcal{S}_{\alpha}^{*}}\left(\mathcal{T}_{3}\right)=(1-\alpha) /\left(\sqrt{8+2 \alpha-\alpha^{2}}\right)$

Proof. (i) The function defined by $m(r)=\left(4 r^{2}-7 r+2\right) / 2(1-r), 0 \leq r<1$ is a decreasing function. Let $\rho=R_{\mathcal{S}_{\alpha}^{*}}\left(\mathcal{T}_{1}\right)$ is the root of the equation $m(r)=\alpha$. From (2.2), it follows that

$$
\operatorname{Re} \frac{z f^{\prime}(z)}{f(z)} \geq \frac{4 r^{2}-7 r+2}{2(1-r)}=m(r) \geq m(\rho)=\alpha .
$$

This shows that $R_{\mathcal{S}_{\alpha}^{*}}\left(\mathcal{T}_{1}\right)$ is atleast $\rho$. At $z=-R_{\mathcal{S}_{\alpha}^{*}}\left(\mathcal{T}_{1}\right)=-\rho$, the function $f_{1}$ satisfies

$$
\frac{z f_{1}^{\prime}(z)}{f_{1}(z)}=\frac{4 \rho^{2}-7 \rho+2}{2(1-\rho)}=\alpha .
$$

Thus the result is sharp.

Also,

$$
\left|\frac{z f_{1}^{\prime}(z)}{f_{1}(z)}-1\right|=1-\alpha .
$$

This proves that the radii $R_{\mathcal{S}^{*}(\alpha)}\left(\mathcal{T}_{1}\right)$ and $R_{\mathcal{S}_{\alpha}^{*}}\left(\mathcal{T}_{1}\right)$ are same.

(ii) The function defined by $n(r)=\left(r^{2}-3 r+1\right) /(1-r), 0 \leq r<1$ is a decreasing function. Let $\rho=R_{\mathcal{S}_{\alpha}^{*}}\left(\mathcal{T}_{2}\right)$ is the root of the equation $n(r)=\alpha$. From (2.4), it follows that

$$
\operatorname{Re} \frac{z f^{\prime}(z)}{f(z)} \geq \frac{1-3 r+r^{2}}{1-r}=n(r) \geq n(\rho)=\alpha .
$$

This shows that $R_{\mathcal{S}_{\alpha}^{*}}\left(\mathcal{T}_{2}\right)$ is atleast $\rho$. At $z=-R_{\mathcal{S}_{\alpha}^{*}}\left(\mathcal{T}_{2}\right)=-\rho$, the function $f_{2}$ satisfies

$$
\frac{z f_{2}^{\prime}(z)}{f_{2}(z)}=\frac{1-3 \rho+\rho^{2}}{1-\rho}=\alpha .
$$

Also, for the function $f_{2}$ we have

$$
\left|\frac{z f_{2}^{\prime}(z)}{f_{2}(z)}-1\right|=1-\alpha .
$$

Hence, the radii $R_{\mathcal{S}^{*}(\alpha)}$ and $R_{\mathcal{S}_{\alpha}^{*}}$ are same for the class $\mathcal{T}_{2}$.

(iii) The function $s(r)=1-3 r / \sqrt{1+r^{2}}, 0 \leq r<1$ is a decreasing function. Let $\rho=R_{\mathcal{S}_{\alpha}^{*}}\left(\mathcal{T}_{3}\right)$ is the root of the equation $s(r)=\alpha$. From (2.6), it follows 


$$
\operatorname{Re}\left(\frac{z f^{\prime}(z)}{f(z)}\right) \geq 1-\frac{3 r}{\sqrt{1+r^{2}}}=s(r) \geq s(\rho)=\alpha .
$$

This shows that $R_{\mathcal{S}_{\alpha}^{*}}\left(\mathcal{T}_{3}\right)$ is atleast $\rho$. At $z=-R_{\mathcal{S}_{\alpha}^{*}}\left(\mathcal{T}_{3}\right)=-\rho$. For the function $f_{3}(z)$, we have

$$
\frac{z f_{3}^{\prime}(z)}{f_{3}(z)}=1-\frac{3 \rho}{\sqrt{1+\rho^{2}}}=\alpha
$$

Now, considering

$$
\left|\frac{z f^{\prime}(z)}{f(z)}-1\right| \leq \frac{3 r}{\sqrt{1+r^{2}}} \leq 1-\alpha .
$$

The function $f_{3}(z)$ provides sharpness, as

$$
\left|\frac{z f_{3}^{\prime}(z)}{f_{3}(z)}-1\right|=\frac{3 z}{\sqrt{1+z^{2}}}=1-\alpha .
$$

The radii $R_{\mathcal{S}^{*}(\alpha)}$ and $R_{\mathcal{S}_{\alpha}^{*}}$ are same for the class $\mathcal{T}_{3}$.

The function $\varphi_{P A R}: \mathbb{D} \rightarrow \mathbb{C}$ given by

$$
\varphi_{P A R}:=1+\frac{2}{\pi^{2}}\left(\log \frac{1+\sqrt{z}}{1-\sqrt{z}}\right)^{2}, \quad \operatorname{Im} \sqrt{z} \geq 0
$$

maps $\mathbb{D}$ on the parabolic region given by $\varphi_{P A R}(\mathbb{D})=\left\{w=u+i v: v^{2}<2 u-1=\{w\right.$ : $\operatorname{Re} w>|w-1|\}$. The class $\mathcal{S}_{p}^{*}:=\mathcal{S}^{*}\left(\varphi_{P A R}\right)=\left\{f \in \mathcal{A}: z f^{\prime}(z) / f(z) \prec \varphi_{P A R}(z)\right\}$ was introduced by Rønning [14], and is known as the class of parabolic starlike functions. The class $\mathcal{S}_{p}^{*}$ consists of the functions $f \in \mathcal{A}$ satisfying

$$
\operatorname{Re}\left(\frac{z f^{\prime}(z)}{f(z)}\right)>\left|\frac{z f^{\prime}(z)}{f(z)}-1\right|, z \in \mathbb{D} .
$$

Evidently, every parabolic starlike function is also starlike of order $1 / 2$.

Shanmugam and Ravichandran [15] had proved that for $1 / 2<a<3 / 2$, then

$$
\{w:|w-a|<a-1 / 2\} \subset\{w: \operatorname{Re} w>|w-1|\} .
$$

The following result gives the radius of parabolic starlikeness for the classes $\mathcal{T}_{1}, \mathcal{T}_{2}$ and $\mathcal{T}_{3}$.

Corollary 3.2. The following results holds for the class $\mathcal{S}_{p}^{*}$.

(i) $R_{\mathcal{S}_{p}^{*}}\left(\mathcal{T}_{1}\right)=(3-\sqrt{5}) / 4 \approx 0.190983$

(ii) $R_{\mathcal{S}_{p}^{*}}\left(\mathcal{T}_{2}\right)=(5-\sqrt{17}) / 4 \approx 0.219224$

(iii) $R_{\mathcal{S}_{p}^{*}}\left(\mathcal{T}_{3}\right)=1 / \sqrt{35} \approx 0.169031$

Proof. In equation (3.1), putting $a=1$ gives $\mathcal{S}_{1 / 2}^{*} \subset \mathcal{S}_{p}^{*}$. Every parabolic starlike function is also starlike function of order $1 / 2$, whence the inclusion $\mathcal{S}_{1 / 2}^{*} \subset \mathcal{S}_{p}^{*} \subset \mathcal{S}^{*}(1 / 2)$. Therefore, for any class $\mathcal{F}$, readily $R_{\mathcal{S}_{1 / 2}^{*}}(\mathcal{F}) \leq R_{\mathcal{S}_{p}^{*}}(\mathcal{F}) \leq R_{\mathcal{S}^{*}(1 / 2)}(\mathcal{F})$.

When $\mathcal{F}=\mathcal{T}_{i}, i=1,2,3$, Theorem 3.1 gives $R_{\mathcal{S}^{*}(\alpha)}\left(\mathcal{T}_{i}\right)=R_{\mathcal{S}_{\alpha}^{*}}\left(\mathcal{T}_{i}\right)$. This shows that $R_{\mathcal{S}_{1 / 2}^{*}}\left(\mathcal{T}_{i}\right)=R_{\mathcal{S}_{p}^{*}}\left(\mathcal{T}_{i}\right)=R_{\mathcal{S}^{*}(1 / 2)}\left(\mathcal{T}_{i}\right)$. So for $\alpha=1 / 2$, from Theorem 3.1, it follows that $R_{\mathcal{S}_{p}^{*}}\left(\mathcal{T}_{1}\right)=(3-\sqrt{5}) / 4, R_{\mathcal{S}_{p}^{*}}\left(\mathcal{T}_{2}\right)=(5-\sqrt{17}) / 4$ and $R_{\mathcal{S}_{p}^{*}}\left(\mathcal{T}_{3}\right)=1 / \sqrt{35}$ 
(i) For the function $f_{1}(z)=z e^{2 z} \sqrt{1+z}$, at $z=-R_{\mathcal{S}_{p}^{*}}\left(\mathcal{T}_{1}\right)=-\rho$, we have

$$
\operatorname{Re} \frac{z f_{1}^{\prime}(z)}{f_{1}(z)}=\frac{4 \rho^{2}-7 \rho+2}{2(1-\rho)}=\frac{1}{2}=\left|\frac{z f_{1}^{\prime}(z)}{f_{1}(z)}-1\right| .
$$

Thus, $R_{\mathcal{S}_{p}^{*}}\left(\mathcal{T}_{1}\right) \leq(3-\sqrt{5}) / 4$.

(ii) For the function $f_{2}(z)=z(1+z) e^{z}$, at $z=-R_{\mathcal{S}_{p}^{*}}\left(\mathcal{T}_{2}\right)=-\rho$, we obtain

$$
\operatorname{Re} \frac{z f_{2}^{\prime}(z)}{f_{2}(z)}=\frac{\rho^{2}+3 \rho+1}{(1+\rho)}=\frac{1}{2}=\left|\frac{z f_{2}^{\prime}(z)}{f_{2}(z)}-1\right| .
$$

Thus $R_{\mathcal{S}_{p}^{*}}\left(\mathcal{T}_{2}\right) \leq(5-\sqrt{17}) / 4$.

(iii) For the function $f_{3}(z)=z\left(z+\sqrt{1+z^{2}}\right)^{3}$, at $z=-R_{\mathcal{S}_{p}^{*}}\left(\mathcal{T}_{3}\right)=-\rho$, we have

$$
\operatorname{Re} \frac{z f_{3}^{\prime}(z)}{f_{3}(z)}=1-\frac{3 \rho}{\sqrt{1+\rho^{2}}}=\frac{1}{2}=\left|\frac{z f_{3}^{\prime}(z)}{f_{3}(z)}-1\right| .
$$

This proves that $R_{\mathcal{S}_{p}^{*}}\left(\mathcal{T}_{3}\right) \leq 1 / \sqrt{35}$.

In 2015, Mendiratta et al. [12] introduced the class of starlike functions associated with the exponential function as $\mathcal{S}_{e}^{*}=\mathcal{S}^{*}\left(e^{z}\right)$ and it satisfies the condition $\left|\log z f^{\prime}(z) / f(z)<1\right|$. They had also proved that, for $e^{-1} \leq a \leq\left(e+e^{-1} / 2\right)$,

$$
\left\{w \in \mathbb{C}:|w-a|<a-e^{-1}\right\} \subseteq\{w \in \mathbb{C}:|\log w|<1\} .
$$

Corollary 3.3. The following results hold for the class $\mathcal{S}_{e}^{*}$.

(i) $R_{\mathcal{S}_{e}^{*}}\left(\mathcal{T}_{1}\right)=\left(-2+7 e-\sqrt{4+4 e+17 e^{2}}\right) / 8 e \approx 0.237983$

(ii) $R_{\mathcal{S}_{e}^{*}}\left(\mathcal{T}_{2}\right)=\left(-1+3 e-\sqrt{1-2 e+5 e^{2}}\right) / 2 e \approx 0.267302$

(iii) $R_{\mathcal{S}_{e}^{*}}\left(\mathcal{T}_{3}\right)=\left(1-2 e+e^{2}\right) /\left(-1+2 e+8 e^{2}\right) \approx 0.215546$

Proof. Mendiratta et. al provided the inclusion (3.2), which gives $\mathcal{S}_{1 / e}^{*} \subset \mathcal{S}_{e}^{*}$. It was also shown in [12, Theorem 2.1(i)] that $\mathcal{S}_{e}^{*} \subset \mathcal{S}_{1 / e}^{*}$. Therefore, $\mathcal{S}_{1 / e}^{*} \subset \mathcal{S}_{e}^{*} \subset \mathcal{S}^{*}(1 / e)$, which provides the required radii as a consequence of Theorem 3.1

(i) For the function $f_{1}(z)=z e^{2 z} \sqrt{1+z}$, at $z=-R_{\mathcal{S}_{e}^{*}}\left(\mathcal{T}_{1}\right)=-\rho$, we have

$$
\left|\log \frac{z f_{1}^{\prime}(z)}{f_{1}(z)}\right|=\left|\log \frac{4 \rho^{2}-7 \rho+2}{2(1-\rho)}\right|=1 .
$$

Thus, $R_{\mathcal{S}_{e}^{*}}\left(\mathcal{T}_{1}\right) \leq\left(-2+7 e-\sqrt{4+4 e+17 e^{2}}\right) / 8 e$.

(ii) For the function $f_{2}(z)=z(1+z) e^{z}$, at $z=-R_{\mathcal{S}_{e}^{*}}\left(\mathcal{T}_{2}\right)=-\rho$, we get

$$
\left|\log \frac{z f_{2}^{\prime}(z)}{f_{2}(z)}\right|=\left|\log \frac{1-3 \rho+\rho^{2}}{(1-\rho)}\right|=1 \text {. }
$$

Thus, $R_{\mathcal{S}_{e}^{*}}\left(\mathcal{T}_{2}\right) \leq\left(-1+3 e-\sqrt{1-2 e+5 e^{2}}\right) / 2 e$.

(iii) For the function $f_{3}(z)=z\left(z+\sqrt{1+z^{2}}\right)^{3}$, at $z=-R_{\mathcal{S}_{e}^{*}}\left(\mathcal{T}_{3}\right)=-\rho$, we obtain

$$
\left|\log \frac{z f_{3}^{\prime}(z)}{f_{3}(z)}\right|=\left|\log \left(1-\frac{3 \rho}{\sqrt{1+\rho^{2}}}\right)\right|=1 .
$$

This proves that $R_{\mathcal{S}_{e}^{*}}\left(\mathcal{T}_{3}\right) \leq\left(1-2 e+e^{2}\right) /\left(-1+2 e+8 e^{2}\right)$. 
Sharma et. al [17] studied the various properties of the class $\mathcal{S}_{c}^{*}=\mathcal{S}^{*}\left(1+(4 / 3) z+(2 / 3) z^{2}\right.$. This class consists the functions $f$ such that $z f^{\prime}(z) / f(z)$ lies in the region bounded by the cardioid $\Omega_{c}=\left\{u+i v:\left(9 u^{2}+9 v^{2}-18 u+5\right)^{2}-16\left(9 u^{2}+9 v^{2}-6 u+1\right)=0\right\}$. They proved the result that, for $1 / 3<a \leq 5 / 3$

$$
\{w \in \mathbb{C}:|w-a|<(3 a-1) / 3\} \subseteq \Omega_{c} .
$$

Various results related to this class are investigated in these papers [13, 16, 18, 19]. Following corollary provides the radius of cardioid starlikeness for each class $\mathcal{T}_{1}, \mathcal{T}_{2}$ and $\mathcal{T}_{3}$.

Corollary 3.4. The following result holds for the class $\mathcal{S}_{c}^{*}$.

(i) $R_{\mathcal{S}_{c}^{*}}\left(\mathcal{T}_{1}\right)=1 / 4=0.25$

(ii) $R_{\mathcal{S}_{c}^{*}}\left(\mathcal{T}_{2}\right)=(4-\sqrt{10}) / 3 \approx 0.279241$

(iii) $R_{\mathcal{S}_{c}^{*}}\left(\mathcal{T}_{3}\right)=2 / \sqrt{77} \approx 0.227921$

Proof. Equation (3.3) provides the inclusion $\mathcal{S}_{1 / 3}^{*} \subset \mathcal{S}_{c}^{*}$ for $a=1$. Thus $R_{\mathcal{S}_{1 / 3}^{*}}\left(\mathcal{T}_{i}\right) \leq R_{\mathcal{S}_{c}^{*}}\left(\mathcal{T}_{i}\right)$ for $i=1,2,3$. The proof is completed by demonstrating $R_{\mathcal{S}_{c}^{*}}\left(\mathcal{T}_{i}\right) \leq R_{\mathcal{S}_{1 / 3}^{*}}\left(\mathcal{T}_{i}\right)$ for $i=1,2,3$.

(i) For the function $f_{1}(z)=z e^{2 z} \sqrt{1+z}$, at $z=-R_{\mathcal{S}_{c}^{*}}\left(\mathcal{T}_{1}\right)=-\rho$, we obtain

$$
\left|\frac{z f_{1}^{\prime}(z)}{f_{1}(z)}\right|=\left|\frac{4 \rho^{2}-7 \rho+2}{2(1-\rho)}\right|=\frac{1}{3} \text {. }
$$

Thus, $R_{\mathcal{S}_{c}^{*}}\left(\mathcal{T}_{1}\right) \leq 1 / 4$.

(ii) For the function $f_{2}(z)=z(1+z) e^{z}$, at $z=-R_{\mathcal{S}_{c}^{*}}\left(\mathcal{T}_{2}\right)=-\rho$, we have

$$
\left|\frac{z f_{2}^{\prime}(z)}{f_{2}(z)}\right|=\left|\frac{1-3 \rho+\rho^{2}}{(1-\rho)}\right|=\frac{1}{3} .
$$

Thus, $R_{\mathcal{S}_{c}^{*}}\left(\mathcal{T}_{2}\right) \leq(4-\sqrt{10}) / 3$.

(iii) For the function $f_{3}(z)=z\left(z+\sqrt{1+z^{2}}\right)^{3}$, at $z=-R_{\mathcal{S}_{c}^{*}}\left(\mathcal{T}_{3}\right)=-\rho$,

$$
\left|\frac{z f_{3}^{\prime}(z)}{f_{3}(z)}\right|=\left|1-\frac{3 \rho}{\sqrt{1+\rho^{2}}}\right|=\frac{1}{3} \text {. }
$$

This proves that $R_{\mathcal{S}_{c}^{*}}\left(\mathcal{T}_{3}\right) \leq 2 / \sqrt{77}$.

In 2019, Cho et al. [5] considered the class of starlike functions associated with sine function. The class $\mathcal{S}_{\text {sin }}^{*}$ is defined as $\mathcal{S}_{\sin }^{*}=\left\{f \in \mathcal{A}: z f^{\prime}(z) / f(z) \prec 1+\sin z:=q_{0}(z)\right\}$ for $z \in \mathbb{D}$. For $|a-1| \leq \sin 1$, the following inclusion holds

$$
\{w \in \mathbb{C}:|w-a|<\sin 1-|a-1|\} \subseteq \Omega_{s} .
$$

Here $\Omega_{s}:=q_{0}(\mathbb{D})$ is the image of the unit disk $\mathbb{D}$ under the mapping $q_{0}(z)=1+\sin z$.

We find the $\mathcal{S}_{\text {sin }}^{*}$ radius for the classes $\mathcal{T}_{1}, \mathcal{T}_{2}$ and $\mathcal{T}_{3}$.

Corollary 3.5. The following results hold for the class $\mathcal{S}_{\text {sin }}^{*}$.

(i) $R_{\mathcal{S}_{\text {sin }}^{*}}\left(\mathcal{T}_{1}\right)=\left(5+2 \sin 1-\sqrt{25-12 \sin 1+4 \sin 1^{2}}\right) / 8 \approx 0.308961$

(ii) $R_{\mathcal{S}_{\text {sin }}^{*}}\left(\mathcal{T}_{2}\right)=\left(2+\sin 1-\sqrt{4+\sin 1^{2}}\right) / 2 \approx 0.335831$

(iii) $R_{\mathcal{S}_{\text {sin }}^{*}}\left(\mathcal{T}_{3}\right)=\sin 1 / \sqrt{9-\sin 1^{2}} \approx 0.292221$ 
Proof. By putting $a=1$ in equation (3.4) we obtain the inclusion $\mathcal{S}_{1-\sin 1}^{*} \subset \mathcal{S}_{\sin }^{*}$. Thus $R_{\mathcal{S}_{1-\sin 1}^{*}} \leq R_{\mathcal{S}_{\text {sin }}^{*}}$ for $i=1,2,3$. The proof is completed by demonstrating $R_{\mathcal{S}_{1-\sin 1}^{*}} \leq R_{\mathcal{S}_{\text {sin }}^{*}}$ for $i=1,2,3$. ( $i)$ For the function $f_{1}(z)=z e^{2 z} \sqrt{1+z}$, at $z=-R_{\mathcal{S}_{\text {sin }}^{*}}\left(\mathcal{T}_{1}\right)=-\rho$, we get

$$
\left|\frac{z f_{1}^{\prime}(z)}{f_{1}(z)}\right|=\left|\frac{4 \rho^{2}-7 \rho+2}{2(1-\rho)}\right|=1+\sin 1 .
$$

Thus, $R_{\mathcal{S}_{\text {sin }}^{*}}\left(\mathcal{T}_{1}\right) \leq\left(5+2 \sin 1-\sqrt{25-12 \sin 1+4 \sin 1^{2}}\right) / 8$.

(ii) For the function $f_{2}(z)=z(1+z) e^{z}$, at $z=-R_{\mathcal{S}_{\sin }^{*}}\left(\mathcal{T}_{2}\right)=-\rho$, we have

$$
\left|\frac{z f_{2}^{\prime}(z)}{f_{2}(z)}\right|=\left|\frac{1-3 \rho+\rho^{2}}{(1-\rho)}\right|=1+\sin 1 .
$$

Thus, $R_{\mathcal{S}_{\text {sin }}^{*}}\left(\mathcal{T}_{2}\right) \leq\left(2+\sin 1-\sqrt{4+\sin 1^{2}}\right) / 2$.

(iii) For the function $f_{3}(z)=z\left(z+\sqrt{1+z^{2}}\right)^{3}$, at $z=-R_{\mathcal{S}_{\sin }^{*}}\left(\mathcal{T}_{3}\right)=-\rho$, we get

$$
\left|\frac{z f_{3}^{\prime}(z)}{f_{3}(z)}\right|=\left|1-\frac{3 \rho}{\sqrt{1+\rho^{2}}}\right|=1+\sin 1 .
$$

This proves that $R_{\mathcal{S}_{\text {sin }}^{*}}\left(\mathcal{T}_{3}\right) \leq \sin 1 / \sqrt{9-\sin 1^{2}}$.

In the next result, we find the radius for starlike functions accociated with a rational function. Kumar and Ravichandran [8] introduced the class of starlike functions associated with a rational function, $\left.\psi(z)=1+\left(z^{2} k+z^{2}\right) /\left(k^{2}-k z\right)\right)$ where $k=\sqrt{2}+1$, defined by $\mathcal{S}_{R}^{*}=\mathcal{S}^{*}(\psi(z))$. For $2(\sqrt{2}-1)<a \leq \sqrt{2}$, they had proved that

$$
\{w \in \mathbb{C}:|w-a|<a-2(\sqrt{2}-1)\} \subseteq \psi(\mathbb{D}) .
$$

Corollary 3.6. The following results holds for the class $\mathcal{S}_{R}^{*}$.

(i) $R_{\mathcal{S}_{R}^{*}}\left(\mathcal{T}_{1}\right)=(11-4 \sqrt{2}-\sqrt{57-24 \sqrt{2}}) / 8 \approx 0.0676475$

(ii) $R_{\mathcal{S}_{R}^{*}}\left(\mathcal{T}_{2}\right)=(5-2 \sqrt{2}-\sqrt{21-12 \sqrt{2}}) / 2 \approx 0.0821135$

(iii) $R_{\mathcal{S}_{R}^{*}}\left(\mathcal{T}_{3}\right)=(\sqrt{-38+27 \sqrt{2}}) / 2 \sqrt{14} \approx 0.0572847$

Proof. For $a=1$ equation (3.5) gives the inclusion $\mathcal{S}_{2(\sqrt{2}-1)}^{*} \subset \mathcal{S}_{R}^{*}$. Thus $R_{\mathcal{S}_{2(\sqrt{2}-1)}^{*}}\left(\mathcal{T}_{i}\right) \leq$ $R_{\mathcal{S}_{R}^{*}}\left(\mathcal{T}_{i}\right)$ for $i=1,2,3$. We next show that $R_{\mathcal{S}_{R}^{*}}\left(\mathcal{T}_{i}\right) \leq R_{\mathcal{S}_{2(\sqrt{2}-1)}^{*}}\left(\mathcal{T}_{i}\right)$ for $i=1,2,3$.

(i) For the function $f_{1}(z)=z e^{2 z} \sqrt{1+z}$, at $z=-R_{\mathcal{S}_{R}^{*}}\left(\mathcal{T}_{1}\right)=-\rho$, we get

$$
\left|\frac{z f_{1}^{\prime}(z)}{f_{1}(z)}\right|=\left|\frac{4 \rho^{2}-7 \rho+2}{2(1-\rho)}\right|=2(\sqrt{2}-1) .
$$

Thus, $R_{\mathcal{S}_{R}^{*}}\left(\mathcal{T}_{1}\right) \leq(11-4 \sqrt{2}-\sqrt{57-24 \sqrt{2}}) / 8$.

(ii) For the function $f_{2}(z)=z(1+z) e^{z}$, at $z=-R_{\mathcal{S}_{R}^{*}}\left(\mathcal{T}_{2}\right)=-\rho$, we have

$$
\left|\frac{z f_{2}^{\prime}(z)}{f_{2}(z)}\right|=\left|\frac{1-3 \rho+\rho^{2}}{(1-\rho)}\right|=2(\sqrt{2}-1) .
$$

Thus, $R_{\mathcal{S}_{R}^{*}}\left(\mathcal{T}_{2}\right) \leq(5-2 \sqrt{2}-\sqrt{21-12 \sqrt{2}}) / 2$. 
(iii) For the function $f_{3}(z)=z\left(z+\sqrt{1+z^{2}}\right)^{3}$, at $z=-R_{\mathcal{S}_{R}^{*}}\left(\mathcal{T}_{3}\right)=-\rho$, we obtain

$$
\left|\frac{z f_{3}^{\prime}(z)}{f_{3}(z)}\right|=\left|1-\frac{3 \rho}{\sqrt{1+\rho^{2}}}\right|=2(\sqrt{2}-1) .
$$

Thus, $R_{\mathcal{S}_{R}^{*}}\left(\mathcal{T}_{3}\right) \leq(\sqrt{-38+27 \sqrt{2}}) / 2 \sqrt{14}$.

In 2020, Wani and Swaminathan [20] introduced the class $\mathcal{S}_{N e}^{*}=\mathcal{S}^{*}\left(1+z-\left(z^{3} / 3\right)\right)$ that maps open disc $\mathbb{D}$ onto the interior of a two cusped kidney shaped curve $\Omega_{N e}:=\{u+i v$ : $\left.\left((u-1)^{2}+v^{2}-4 / 9\right)^{3}-4 v^{2} / 3<0\right\}$. For $1 / 3<a \leq 1$, they had proved that

$$
\{w \in \mathbb{C}:|w-a|<a-1 / 3\} \subseteq \Omega_{N e} .
$$

Our next theorem determines the $\mathcal{S}_{N e}^{*}$ radius results for the classes $\mathcal{T}_{1}, \mathcal{T}_{2}$ and $\mathcal{T}_{3}$.

Corollary 3.7. The following results hold for the class $\mathcal{S}_{N e}^{*}$.

(i) $R_{\mathcal{S}_{N e}^{*}}\left(\mathcal{T}_{1}\right)=1 / 4=0.25$

(ii) $R_{\mathcal{S}_{N e}^{*}}\left(\mathcal{T}_{2}\right)=(4-\sqrt{10}) / 3 \approx 0.279241$

(iii) $R_{\mathcal{S}_{N e}^{*}}\left(\mathcal{T}_{3}\right)=2 / \sqrt{77} \approx 0.227921$

Proof. From equation (3.6) we obtain the inclusion $\mathcal{S}_{1 / 3}^{*} \subset \mathcal{S}_{N e}^{*}$ for $a=1$. This shows that $R_{\mathcal{S}_{1 / 3}^{*}}\left(\mathcal{T}_{i}\right) \leq R_{\mathcal{S}_{N e}^{*}}\left(\mathcal{T}_{i}\right)$ for $i=1,2,3$.

(i) For the function $f_{1}(z)=z e^{2 z} \sqrt{1+z}$, at $z=-R_{\mathcal{S}_{N e}^{*}}\left(\mathcal{T}_{1}\right)=-\rho$,

$$
\left|\frac{z f_{1}^{\prime}(z)}{f_{1}(z)}\right|=\left|\frac{4 \rho^{2}-7 \rho+2}{2(1-\rho)}\right|=\frac{1}{3} \text {. }
$$

Thus, $R_{\mathcal{S}_{N e}^{*}}\left(\mathcal{T}_{1}\right) \leq 1 / 4$.

(ii) For the function $f_{2}(z)=z(1+z) e^{z}$, at $z=-R_{\mathcal{S}_{R}^{*}}\left(\mathcal{T}_{2}\right)=-\rho$,

$$
\left|\frac{z f_{2}^{\prime}(z)}{f_{2}(z)}\right|=\left|\frac{1-3 \rho+\rho^{2}}{(1-\rho)}\right|=\frac{1}{3} .
$$

Thus, $R_{\mathcal{S}_{N e}^{*}}\left(\mathcal{T}_{2}\right) \leq(4-\sqrt{10}) / 3$.

(iii) For the function $f_{3}(z)=z\left(z+\sqrt{1+z^{2}}\right)^{3}$, at $z=-R_{\mathcal{S}_{N e}^{*}}\left(\mathcal{T}_{3}\right)=-\rho$,

$$
\left|\frac{z f_{3}^{\prime}(z)}{f_{3}(z)}\right|=\left|1-\frac{3 \rho}{\sqrt{1+\rho^{2}}}\right|=\frac{1}{3} .
$$

This proves that $R_{\mathcal{S}_{N e}^{*}}\left(\mathcal{T}_{3}\right) \leq 2 / \sqrt{77}$.

Goel and Kumar [7] introduced the class $\mathcal{S}_{S G}^{*}:=\mathcal{S}^{*}\left(2 / 1+e^{-z}\right)$, where $2 /\left(1+e^{-z}\right)$ is a modified sigmoid function that maps $\mathbb{D}$ onto the region $\Omega_{S G}:=\{w=u+i v: \mid \log (w /(2-$ $w)) \mid<1\}$. Precisely, $f \in \mathcal{S}_{S G}^{*}$ provided the function $z f^{\prime}(z) / f(z)$ maps $\mathbb{D}$ onto the region lying inside the domain $\Omega_{S G}$. For $2 /(e+1)<a<2 e /(1+e)$, Goel and Kumar [7] had proved the following inclusion

$$
\left\{w \in \mathbb{C}:|w-a|<r_{S G}\right\} \subset \Omega_{S G},
$$

provided $r_{S G}=((e-1) /(e+1))-|a-1|$. Next result is about the $\mathcal{S}_{S G}^{*}$ radius for the defined classes. 
Theorem 3.8. The following results hold for the class $\mathcal{S}_{S G}^{*}$.

(i) $R_{\mathcal{S}_{S G}^{*}}\left(\mathcal{T}_{1}\right)=\left(3+7 e-\sqrt{41+42 e+17 e^{2}}\right) /(8+8 e) \approx 0.177213$

(ii) $R_{\mathcal{S}_{S G}^{*}}\left(\mathcal{T}_{2}\right)=\left(1+3 e-\sqrt{5+6 e+5 e^{2}}\right)(2+2 e) \approx 0.204712$

(iii) $R_{\mathcal{S}_{S G}^{*}}\left(\mathcal{T}_{3}\right)=\left(\sqrt{1-2 e+e^{2}}\right) /\left(8+20 e+8 e^{2}\right) \approx 0.1559$

Proof. (i) The function defined by $m(r)=\left(4 r^{2}-7 r+2\right) / 2(1-r), 0 \leq r<1$ is a decreasing function. Let $\rho=R_{\mathcal{S}_{S G}^{*}}\left(\mathcal{T}_{1}\right)$ is the root of the equation $m(r)=2 /(1+e)$. For $0<r \leq$ $R_{\mathcal{S}_{S G}^{*}}\left(\mathcal{T}_{1}\right)$, we have $m(r) \geq 2 /(1+e)$. That is

$$
\frac{r(5-4 r)}{2(1-r)} \leq \frac{e-1}{e+1}
$$

For the class $\mathcal{T}_{1}$, the centre of the disk is 1 , therefore the disk obtained in (2.2) is contained in the region bounded by modified sigmoid, by equation (3.7). For the function $f_{1}(z)=$ $z e^{2 z} \sqrt{1+z}$, at $z=-R_{\mathcal{S}_{S G}^{*}}\left(\mathcal{T}_{1}\right)=-\rho$, we have

$$
\left|\log \frac{z f_{1}^{\prime}(z) / f_{1}(z)}{2-\left(z f_{1}^{\prime}(z) / f_{1}(z)\right)}\right|=\left|\frac{\left(4 \rho^{2}-7 \rho+2\right) / 2(1-\rho)}{2-\left(\left(4 \rho^{2}-7 \rho+2\right) / 2(1-\rho)\right)}\right|=1 .
$$

(ii) The function defined $n(r)=\left(r^{2}-3 r+1\right) /(1-r), 0 \leq r<1$ is a decreasing function. Let $\rho=R_{\mathcal{S}_{S G}^{*}}\left(\mathcal{T}_{2}\right)$ is the root of the equation $n(r)=1 / 3$. For $0<r \leq R_{\mathcal{S}_{S G}^{*}}\left(\mathcal{T}_{2}\right)$, we have $n(r) \geq 2 / 1+e$. That is,

$$
\frac{r(-2+r)}{(-1+r)} \leq \frac{e-1}{e+1}
$$

For the class $\mathcal{T}_{2}$, the centre of the disk is 1 , therefore the disk obtained in (2.4) is contained in the region bounded by the modified sigmoid, using equation (3.7). For the function $f_{2}(z)=z(1+z) e^{z}$, at $z=-R_{\mathcal{S}_{S G}^{*}}\left(\mathcal{T}_{3}\right)=-\rho$,

$$
\left|\log \frac{z f_{2}^{\prime}(z) / f_{2}(z)}{2-\left(z f_{2}^{\prime}(z) / f_{2}(z)\right)}\right|=\left|\frac{\left(1-3 \rho+\rho^{2}\right) /(1-\rho)}{2-\left(\left(1-3 \rho+\rho^{2}\right) /(1-\rho)\right)}\right|=1 .
$$

(iii) The function defined $s(r)=1-3 r / \sqrt{1+r^{2}}, 0 \leq r<1$ is a decreasing function. Let $\rho=R_{\mathcal{S}_{S G}^{*}}\left(\mathcal{T}_{3}\right)$ is the root of the equation $s(r)=2 /(1+e)$. For $0<r \leq R_{\mathcal{S}_{S G}^{*}}\left(\mathcal{T}_{3}\right)$, we have $s(r) \geq 2 / 1+e$. That is

$$
\frac{3 r}{\sqrt{1+r^{2}}} \leq \frac{e-1}{e+1}
$$

For the class $\mathcal{T}_{3}$, the centre of the disk is 1 , therefore the disk obtained in (2.6) is contained in the region bounded by the modified sigmoid, by equation (3.7). For the function $f_{2}(z)=$ $z\left(z+\sqrt{1+z^{2}}\right)^{3}$, at $z=-R_{\mathcal{S}_{S G}^{*}}\left(\mathcal{T}_{3}\right)=-\rho$,

$$
\left|\log \frac{z f_{3}^{\prime}(z) / f_{3}(z)}{2-\left(z f_{3}^{\prime}(z) / f_{3}(z)\right)}\right|=\left|\frac{1-(3 \rho) / \sqrt{1+\rho^{2}}}{2-\left(1-(3 \rho) /\left(\sqrt{1+\rho^{2}}\right)\right)}\right|=1 .
$$




\section{REFERENCES}

[1] R. M. Ali, N. K. Jain and V. Ravichandran, Radii of starlikeness associated with the lemniscate of Bernoulli and the left-half plane, Appl. Math. Comput. 218 (2012), no. 11, 6557-6565.

[2] R. M. Ali, N. K. Jain and V. Ravichandran, On the radius constants for classes of analytic functions, Bull. Malays. Math. Sci. Soc. (2) 36 (2013), no. 1, 23-38.

[3] R. M. Ali, K. Sharma and V. Ravichandran, Starlikeness of analytic functions with subordinate ratios, preprint.

[4] S. Afis and K. I. Noor, On subclasses of functions with boundary and radius rotations associated with crescent domains, Bull. Korean Math. Soc. 57 (2020), no. 6, 1529-1539.

[5] N. E. Cho, V. Kumar, S. S. Kumar and V. Ravichandran, Radius problems for starlike functions associated with the sine function, Bull. Iranian Math. Soc. 45 (2019), no. 1, 213-232.

[6] S. Gandhi and Ravichandran, V. Starlike functions associated with a lune. Asian-Eur. J. Math. 10 (2017), no. 4, 1750064, 12 pp.

[7] P. Goel and S. Sivaprasad Kumar, Certain class of starlike functions associated with modified sigmoid function, Bull. Malays. Math. Sci. Soc. 43 (2020), no. 1, 957-991.

[8] S. Kumar and V. Ravichandran, A subclass of starlike functions associated with a rational function, Southeast Asian Bull. Math. 40 (2016), no. 2, 199-212.

[9] T. H. MacGregor, The radius of univalence of certain analytic functions, Proc. Amer. Math. Soc. 14 (1963), 514-520.

[10] T. H. MacGregor, The radius of univalence of certain analytic functions. II, Proc Amer. Math. Soc. 14 (1963), 521-524.

[11] T. H. MacGregor, A class of univalent functions, Proc. Amer. Math. Soc. 15 (1964), 311-317.

[12] R. Mendiratta, S. Nagpal and V. Ravichandran, On a subclass of strongly starlike functions associated with exponential function, Bull. Malays. Math. Sci. Soc. 38 (2015), no. 1, 365-386.

[13] V. Ravichandran and K. Sharma, Sufficient conditions for starlikeness, J. Korean Math. Soc. 52 (2015), no. $4,727-749$.

[14] F. Rønning, Uniformly convex functions and a corresponding class of starlike functions, Proc. Amer. Math. Soc. 118 (1993), no. 1, 189-196.

[15] T. N. Shanmugam and V. Ravichandran, Certain properties of uniformly convex functions, Computational Methods And Function Theory 1994 (Penang), 319-324, Ser. Approx. Decompos., 5 World Sci. Publ., River Edge, NJ.

[16] K. Sharma, N. E. Cho and V. Ravichandran, Sufficient conditions for strong starlikeness, Bull. Iranian Math. Soc. (2020), DOI:10.1007/s41980-020-00452-z.

[17] K. Sharma, N. K. Jain and V. Ravichandran, Starlike functions associated with a cardioid, Afr. Mat. (Springer) 27 (2016), no. 5, 923-939.

[18] K. Sharma and V. Ravichandran, Applications of subordination theory to starlike functions, Bull. Iranian Math. Soc. 42 (2016), no. 3, 761-777.

[19] K. Sharma and V. Ravichandran, Sufficient conditions for Janowski starlike functions, Stud. Univ. Babeş-Bolyai Math. 61 (2016), no. 1, 63-76.

[20] L. A. Wani and A. Swaminathan, Starlike and convex functions associated with a nephroid domain, Bull. Malays. Math. Sci. Soc. (2020). https://doi.org/10.1007/s40840-020-00935-6

Department of Mathematics, National Institute of Technology, Tiruchirappalli-620 015, INDIA

Email address: shaluyadav903@gmail.com

Department of Mathematics, Atma Ram Sanatan Dharma College, University of Delhi, DELHI-110 021, INDIA

Email address: ksharma@arsd.du.ac.in; kanika.divika@gmail.com

Department of Mathematics, National Institute of Technology, Tiruchirappalli-620 015 , INDIA

Email address: ravic@nitt.edu; vravi68@gmail.com 